\title{
SILICA MONOLITHIC CAPILLARY COLUMNS FOR HILIC SEPARATIONS
}

\author{
Dana MORaVcová*1 AND JOSEF PLANETA ${ }^{1}$ \\ ${ }^{1}$ Institute of Analytical Chemistry of the Czech Academy of Sciences, Veveri 97, 60200 Brno, CZECH \\ REPUBLIC
}

\begin{abstract}
The monolithic capillary columns $(0.1 \mathrm{~mm} \times 150 \mathrm{~mm})$ prepared by the acidic hydrolysis of tetramethoxysilane (TMOS) in the presence of polyethylene glycol (PEG) and urea were modified by zwitterionic stationary phases and evaluated under HILIC separation conditions by employing a mixture containing nucleosides and nucleotides. The polymeric layer of zwitterions did not affect the high separation efficiency of the original silica monolith. The prepared zwitterionic columns exhibited high separation efficiencies in a range $61,000-289,000$ theoretical plates $/ \mathrm{m}$ for a 2methacryloyloxyethyl phosphorylcholine-based stationary phase and in a range 59,000-135,000 theoretical plates/m for a [2-(methacryloyloxy)ethyl]dimethyl-(3-sulfopropyl)ammonium hydroxide-based stationary phase under optimal separation conditions. The grafted layer of zwitterions on the silica monolithic surface also significantly improved the separation selectivity to compounds of interest.
\end{abstract}

Keywords: HILIC, silica monolith, nucleoside, nucleotide, capillary chromatography

\section{Introduction}

Silica-based monolithic columns were developed by Nakanishi and co-workers in the early 1990s [1,2]. The preparation procedure of the columns has been improved over the years [3]. The unique structure of silica-based monoliths facilitates a high degree of permeability of separation beds and good mass transfer kinetics leading to a high separation efficiency. Thus, liquid chromatography has been identified as an alternative to particle packed columns.

While silica-based monolithic columns dedicated to reversed-phase separations are well established, the new stationary phases of hydrophilic interaction chromatography (HILIC) separation are still a focus of research. Concerning the published papers, the preparation of silicabased monoliths via the so-called "one-pot" process prevails. On the other hand, the chemical modification of preformed silica monoliths offers a well-defined silica skeleton of almost the same permeability and separation efficiency for all designed stationary phases.

This contribution presents a characterization of silica-based monolithic capillary columns prepared by employing the second approach. The zwitterionic monomers [2-(methacryloyloxy)ethyl]dimethyl-(3sulfopropyl)ammonium hydroxide (MEDSA) and 2methacryloyloxyethyl phosphorylcholine (MEPC) were used to prepare the desired stationary phases. The prepared columns were evaluated under HILIC separation

\footnotetext{
*Correspondence: moravcova@iach.cz
}

conditions by employing a mixture containing nucleosides and nucleotides.

\section{Experimental}

\subsection{Material and Instrumentation}

MEDSA and MEPC monolithic capillary columns were prepared following a protocol outlined in our previous study [4]. In order to obtain a stable polymeric layer of MEPC on the silica-based monolith, the polymerization time was prolonged to 6 hours.

Toluene, adenosine, uridine, cytidine and nucleotides - adenosine monophosphate (AMP), adenosine diphosphate (ADP), adenosine triphosphate (ATP), cytidine monophosphate (CMP), cytidine diphosphate (CDP), uridine monophosphate (UMP), and uridine diphosphate (UDP) - were purchased from Sigma-Aldrich (Prague, Czech Republic). Acetic acid, ammonium hydroxide and acetonitrile (ACN) (CHROMASOLV, HPLC gradient grade) were obtained from Riedel-de Haën (Seelze, Germany).

High-performance liquid chromatography (HPLC) equipment consisted of two identical syringe pumps (100DM model with a D-series controller, Teledyne ISCO, Lincoln, Nebraska, USA) directly connected to a static nano mixer (60 nl volume) (Upchurch Scientific, Oak Harbor, Washington, USA). The injection was performed by an electrically actuated E90-220 injection valve with a $60 \mathrm{nl}$ internal loop (Valco Instruments, Houston, Texas, USA) and a T-splitter with a restrictor (fused 


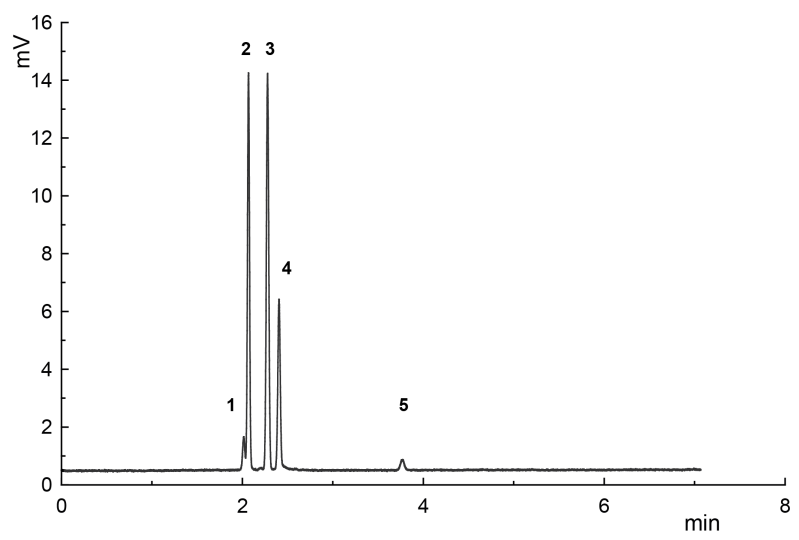

Figure 1: HILIC separation of four purine bases in a bare silica monolithic capillary column. Mobile phase: $95 \%$ (v/v) $\mathrm{ACN}, 5 \%(\mathrm{v} / \mathrm{v}) 100 \mathrm{mM}$ ammonium acetate $\mathrm{pH}=4.5$; flow rate $500 \mathrm{~nL} / \mathrm{min}$; UV detection at $210 \mathrm{~nm}$. Peak identification: 1 - toluene (unretained compound), 2 - xanthine, 3 - caffeine, 4 - theobromine, and 5 -hypoxanthine.

silica capillary $0.025 \mathrm{~mm} \times 150 \mathrm{~mm}$ in length). The inlet section of the monolithic column was inserted through the body of the splitter into the injection valve to minimize extra-column dispersion.

The outlet of the monolithic column was connected to a Spectra $100 \mathrm{UV} / \mathrm{Vis}$ detector (Thermo Separation Products, Waltham, Massachusetts, USA) via a $0.035 \mathrm{~mm}$ $\times 120 \mathrm{~mm}$-long fused silica capillary (with an optical bubble cell window inner diameter (ID) $0.110 \mathrm{~mm}$ ) using a polytetrafluoroethylene (PTFE) sleeve. UV detection was performed at $210 \mathrm{~nm}$. The detector signal was processed by the chromatography station software Clarity (DataApex, Prague, Czech Republic).

\section{Results and Discussion}

\subsection{Chromatographic properties of the bare silica monolith}

The prepared bare silica monolithic capillary columns were evaluated under HILIC conditions by employing a mixture containing toluene as an unretained compound and four purine bases, namely xanthine, caffeine, theobromine and hypoxanthine. The obtained chromatogram

Table 1: The retention factor $(k)$, the height equivalent to the theoretical plate $(H)$, the number of theoretical plates per meter $(N)$, and peak asymmetry $(A s)$ obtained on the silica monolithic column. See Fig. 1 for separation conditions.

\begin{tabular}{l|c|c|c|c}
\hline Compound & $k$ & $H / \mu \mathrm{m}$ & $N$ & $A s$ \\
\hline Toluene & - & 7.4 & 135,185 & 0.67 \\
Xanthine & 0.03 & 4.5 & 222,547 & 0.88 \\
Caffeine & 0.13 & 5.8 & 172,795 & 1.00 \\
Theobromine & 0.19 & 5.2 & 192,528 & 1.13 \\
Hypoxanthine & 0.87 & 4.8 & 209,601 & 1.33 \\
\hline
\end{tabular}

Table 2: The retention factor $(k)$, number of theoretical plates per meter $(N)$, and peak asymmetry $(A s)$ obtained on the MEPC column. See Fig. 2 for the separation conditions.

\begin{tabular}{l|c|c|c}
\hline Compound & $k$ & $N$ & $A s$ \\
\hline Toluene & - & 138,955 & 1.0 \\
Adenosine & 0.16 & 201,048 & 1.0 \\
Uridine & 0.25 & 289,537 & 1.1 \\
Cytidine & 0.41 & 244,314 & 0.9 \\
AMP & 1.24 & 206,697 & 1.3 \\
UMP & 1.66 & 183,465 & 1.5 \\
CMP & 2.50 & 157,760 & 1.4 \\
ADP & 3.98 & 122,169 & 2.0 \\
UDP & 5.34 & 112,389 & 2.4 \\
CDP & 7.58 & 81,476 & 2.8 \\
ATP & 9.73 & 61,462 & 2.1 \\
\hline
\end{tabular}

where all purine bases are baseline separated and have narrow symmetrical peaks is presented in Fig. 1.

The prepared column exhibits a high degree of separation efficiency reaching values of between 135,000 and 220,000 theoretical plates/m which corresponds to the minimum plate height within the range of $4.5-7.4 \mu \mathrm{m}$, as outlined in Table 1.

\subsection{HILIC separation of nucleosides and nu- cleotides}

The commercially available methacrylate-based monomers MEDSA and MEPC were selected for the preparation of zwitterionic stationary phases. The bare silica monolith prepared in a $0.1 \mathrm{~mm}$ ID fused silica capillary was modified by 3-(trimethoxysilyl)propyl methacrylate and a polymer layer, with the appropriate zwitterion grafted onto it. The suitability of these columns for the isocratic HILIC separation of nucleosides and nucleotides was studied.

Chromatograms for selected compounds obtained on MEDSA (Fig. 2A) and MEPC (Fig. 2B) capillary columns are presented in Fig. 2. An enhanced degree of separation was obtained on the MEPC column (Fig. 2B) where all 11 compounds present in a sample mixture were well separated within 25 mins. The value of peak asymmetry $(A s)$ reflects the change in the molecular shape of the analyte and increases slightly as the number of phosphate groups increases in the relevant nucleotide, as presented in Table 2.

On the other hand, the separation efficiency decreases along with the number of phosphate groups in the molecule of nucleotide exhibit lower values of $N$ compared to nucleosides. A similar trend was also observed on the MEDSA-modified monolithic capillary column.

The MEDSA-modified monolithic column exhibits a poorer separation efficiency than the MEPC column for all nucleosides and nucleotides, even the number of theoretical plates per meter for toluene is comparable, as illustrated in Table 3. The MEDSA column also exhibits 

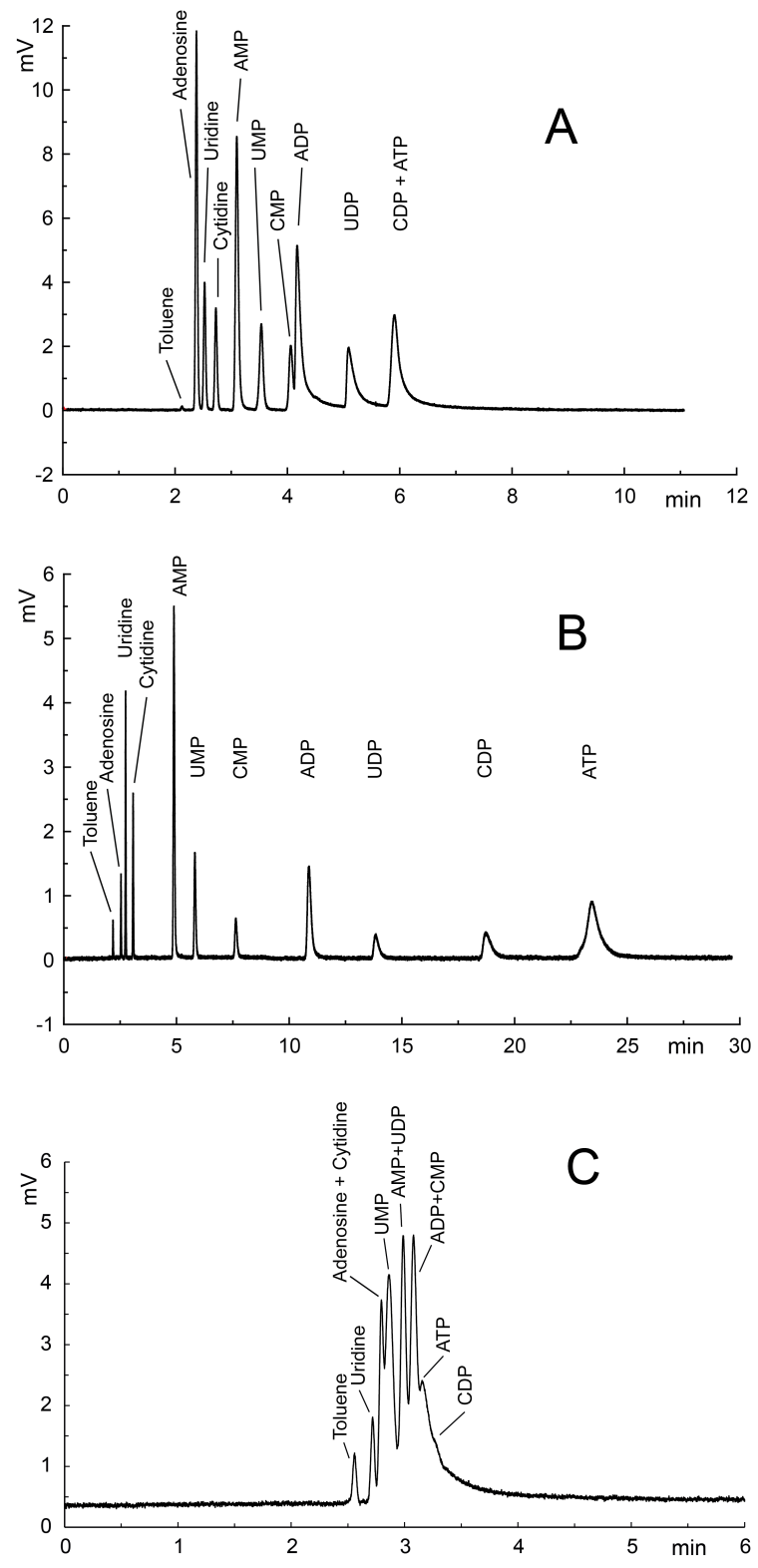

Figure 2: Separation of nucleosides and nucleotides on prepared MEDSA (A), MEPC (B) and bare silica (C) monolithic columns. Mobile phase: $70 \%$ (v/v) ACN/30\% $(\mathrm{v} / \mathrm{v}) 50 \mathrm{mM}$ ammonium acetate $\mathrm{pH}=4.5$; flow rate 500 $\mathrm{nl} / \mathrm{min}$; UV detection at $210 \mathrm{~nm}$.

a lower retention factor for selected compounds, especially for di- and triphosphates of the relevant nucleosides. This can be explained by the fact that monomers used for column modification differ in terms of their terminal groups, which affects the selectivity of the prepared stationary phases. The MEDSA monolithic capillary column contains negatively charged terminal sulfo groups which decrease the retention factor of negatively charged nucleotides. On the other hand, the MEPC monomer provides the stationary phase with a slightly positive charge originating from the phosphorylcholine functional group which improves the retention factor of negatively charged
Table 3: The retention factor $(k)$, number of theoretical plates per meter $(N)$, and peak asymmetry $(A s)$ obtained on the MEDSA column. See Fig. 2 for the separation conditions.

\begin{tabular}{l|c|c|c}
\hline Compound & $k$ & $N$ & $A s$ \\
\hline Toluene & - & 131,000 & 1.2 \\
Adenosine & 0.12 & 135,641 & 1.1 \\
Uridine & 0.19 & 129,895 & 1.2 \\
Cytidine & 0.28 & 130,754 & 1.2 \\
AMP & 0.46 & 101,991 & 1.5 \\
UMP & 0.66 & 89,032 & 1.0 \\
CMP & 0.91 & 90,838 & 1.1 \\
ADP & 0.97 & 71,440 & 1.7 \\
UDP & 1.40 & 48,371 & 5.1 \\
CDP & 1.78 & 49,250 & 3.1 \\
ATP & 1.78 & 59,378 & 3.5 \\
\hline
\end{tabular}

nucleotides under the HILIC separation conditions, e.g. the retention factor $k$ for ATP is approximately 5 times higher on the MEPC column compared to the MEDSA column.

The separation of nucleosides and nucleotides on a bare silica monolithic capillary column is shown in Fig. $2 \mathrm{C}$. The bare silica monolith does not exhibit a sufficient degree of selectivity to compounds of the target. All compounds are eluted from the column within $1 \mathrm{~min}$ and accompanied with the coelution of most compounds.

\section{Conclusion}

A silica-based monolith in a capillary format coated with zwitterionic polymer layers is a good alternative for the analysis of complex mixtures containing highly polar compounds such as nucleosides and nucleotides. In particular, the MEPC stationary phase containing positively charged terminal groups enables the highly selective HILIC separation of nucleosides and nucleotides to be achieved. The utilization of such a stationary phase with regard to the analysis of real samples, where usually other highly polar-interfering species originating from complex matrices are present and complicate the analysis, should be evaluated in the future.

\section{Acknowledgement}

This research was supported by the Czech Science Foundation (Project No. 16-03749S), the Ministry of the Interior of the Czech Republic (Project No. VI20172020069), and by the Czech Academy of Sciences (Institutional Support RVO:68081715).

\section{REFERENCES}

[1] Nakanishi, K., Soga, N.: Phase separation in silica sol-gel system containing polyacrylic acid I. Gel formation behavior and effect of solvent composition, J. Non-Cryst. Solids, 1992139 1-13 DOI: 10.1016/S0022-3093(05)80800-2 
[2] Minakuchi, H., Nakanishi, K., Soga, N., Ishizuka, N., Tanaka, N.: Octadecylsilylated Porous Silica Rods as Separation Media for Reversed-Phase Liquid Chromatography, Anal. Chem., 1996 68(19) 3498-3501 DOI: 10.1021/ac960281m

[3] Ikegami, T., Tanaka, N.: Recent Progress in Monolithic Silica Columns for High-Speed and HighSelectivity Separations, Annu. Rev. Anal. Chem.,
2016 9, 317-342 DOI: 10.1146/annurev-anchem-071114040102

[4] Moravcová, D., Planeta, J., Kahle, V., Roth, M.: Zwitterionic silica-based monolithic capillary columns for isocratic and gradient hydrophilic interaction liquid chromatography, J. Chromatogr. A, 2012 1270, 178-185 DOI: 10.1016/j.chroma.2012.11.005 